\title{
Medium-Term Frequency Stability of Hydrogen Masers as Measured by a Cesium Fountain
}

\author{
T.E. Parker, S.R. Jefferts and T.P. Heavner \\ Time and Frequency Division \\ National Institute of Standards and Technology \\ Boulder, CO 80305, USA \\ tparker@boulder.nist.gov
}

\begin{abstract}
Hydrogen masers are used extensively in time scale ensembles, but the frequency drift must be accurately known. NIST-F1, the cesium fountain primary frequency standard at the National Institute of Standards and Technology, now operates nearly every month and has provided an unprecedented opportunity to characterize the medium-term frequency stability ( $\tau=\mathbf{3 0}$ to 90 days) of six hydrogen masers. An unexpected observation was a moderate level of correlation in the frequency fluctuations of all the masers.
\end{abstract}

\section{INTRODUCTION}

Active, cavity tuned, hydrogen masers are used extensively in time scale ensembles. However, the best results can be achieved only if the significant frequency drift of a hydrogen maser is modeled in the time scale algorithm. This has been done in the maser based ensemble at the National Institute of Standards and Technology (NIST) for over 15 years [1], but accurately determining the maser drift rates in a timely fashion has always been difficult. In order to measure the frequency drift rate, the maser frequency must, ultimately, be compared to a stable and/or accurate frequency standard. Thermal beam primary frequency standards generally operate continuously, but have relatively large uncertainties and noise levels. Consequently it takes many months to obtain a meaningful drift rate. Cesium fountain primary frequency standards have much smaller uncertainties, but initially they operated only occasionally. Thus the problem still existed that the maser drift rates could be determined only over long time intervals. NIST-F1, the cesium fountain primary frequency standard at NIST, now operates almost every month and has provided an unprecedented opportunity to measure the drift rates of all six masers at NIST at intervals of about 40 days with a fractional frequency uncertainty of less than $2 \times 10^{-17} / \mathrm{d}$.

The Bureau International des Poids et Mesures (BIPM) calculates and publishes Coordinated Universal Time (UTC) once a month, and local metrology laboratories use this information to steer their time scales. Thus 30 days is a natural time interval to update maser drift rates if the cesium fountain is not available continuously. In this investigation we operated NIST-F1 approximately once a month. From these runs we could periodically determine the frequency of one of our masers with an uncertainty of about $5 \times 10^{-16}$. By using our time scale measurement system we could then relate the frequency of the measured maser to the frequencies of all the other masers with an additional uncertainty of less than $1 \times 10^{-17}$.

Over the 19 months of this investigation NIST-F1 was operated 15 times with a total average fractional run time of $28 \%$ and an average time interval between runs of 38.9 days. The average run length was approximately 10 days. The primary goals of this study were to acquire a better understanding of the frequency stability of the masers at intervals of about 30 to 60 days and to improve our estimates of the maser frequency drift rates. In addition, this also provided the opportunity to measure with very low uncertainty the frequencies of the hydrogen masers against a fundamentally different type of frequency standard. This is important to help determine whether there are any commonmode frequency fluctuations in the masers that cannot be observed within the maser ensemble. All of NIST's masers are operated in individual environmental chambers that control temperature and relative humidity, but all the masers are also located in close proximity to each other. Five of the six masers are in one room and the sixth is located in another room across a hallway. Common-mode frequency variations have always been a concern even though we have measured the environmental sensitivities of most of the masers, and we monitor temperature, relative humidity, barometric pressure, magnetic field, and power line voltage [2]. The masers used in this study are all active, cavity-tuned masers that were purchased between 1990 and 2005.

\section{LONG-TERM FREQUENCY DRIFT}

The typical frequency drift of a hydrogen maser is large compared to most commercial thermal beam cesium standards and is on the order of $1 \times 10^{-16} /$ day after some initial operating period. Furthermore, the drift is not linear over long periods of time, and significant higher-order components are present. 


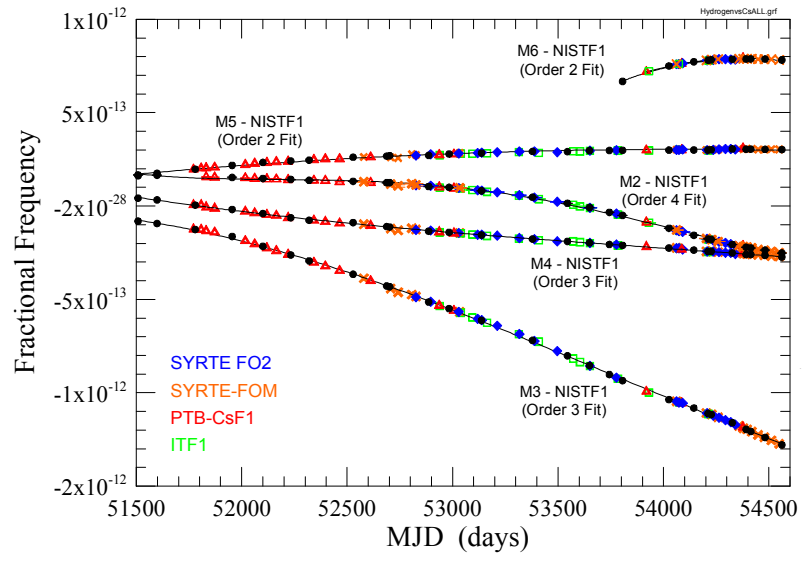

Figure 1. Long-term frequency drift characteristics of hydrogen masers as measured by five primary frequency standards. The solid black lines are least mean square fits to the NIST-F1 data.

Figure 1 shows the fractional frequency drift of five of the NIST masers over a period of about 8.5 years, ending in February 2008. Data referenced to five different cesium fountain primary frequency standards are shown, but the black lines are fit lines to only the NIST-F1 data, which is the only fountain present over the entire time interval of the plot. The horizontal axis is in days using the Modified Julian Date (MJD). It is clear that higher order terms are necessary to fit the data over this long time interval. Generally the maser frequencies have moved independently of each other, but note how the frequencies of M2 and M4 have converged. This will be discussed more in Section III. There are some small arbitrary frequency offsets to each maser because of environmental corrections. Data from maser M1 (the oldest maser) is not shown because it had several significant interruptions during this time interval.

\section{MEDIUM-TERM FREQUENCY STABILITY}

The short-term stability ( $\tau$ from 2 seconds to 24 days) of a hydrogen maser has been previously measured against NISTF1 [3], and the long-term stability for intervals of over one

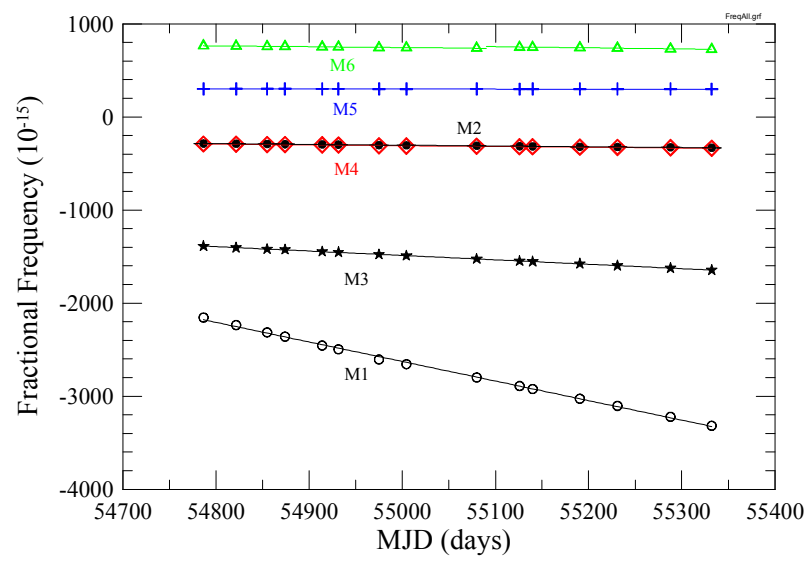

Figure 2. Output frequencies of five masers as measured by NIST-F1. The solid lines are linear least mean square fits. hundreds days to many years has been investigated from data such as that in Fig. 1 [4]. However, the maser stability from about 30 days to 200 days has not been adequately documented by measurements against a primary frequency standard. In this section we discuss the results of such an investigation. Figure 2 shows the measured fractional frequencies offsets (from the $5 \mathrm{MHz}$ or $100 \mathrm{MHz}$ outputs) of the six masers relative to NIST-F1 over an interval of 562 days ending on May 16, 2010. These data cover a time interval beyond that in Fig. 1. The frequency of maser M2 was measured directly by the fountain, and the remaining maser frequencies were measured relative to M2 by using the time scale measurement system. The solid lines are from linear least mean square fits to the 15 data points. Though the frequencies of M2 and M4 appear to be the same, they are actually $4.4 \times 10^{-15}$ apart. Maser M1, which has the largest drift rate, is the oldest maser and has undergone significant repairs that required breaking the vacuum. Because of time and financial constraints the repairs were performed in a less than optimal fashion and the maser now has a significantly larger drift rate than any of the other masers. No environmental corrections were made to this data, or any following data in this paper.

The masers each have a frequency synthesizer that allows the output frequencies to be adjusted. We had deliberately offset the initial output frequencies of the masers so that they were not too close together. From the synthesizer settings the internal frequencies can be calculated and these are shown in Fig. 3. M1 still has the lowest frequency and, as expected, the remaining five are more closely bunched in frequency than in Fig 2. Note that the internal frequencies of M2 and M4 are not as closely spaced as the output frequencies, and that the frequencies of M3 and M5 have actually crossed over each other. An identical arbitrary fixed frequency offset has been applied to all the masers for these data.

\section{A. Frequency Deviations}

The data in Figs. 2 and 3 show the gross frequency characteristics of the masers, but to see the finer details we must look at the frequency deviations from the linear fits.

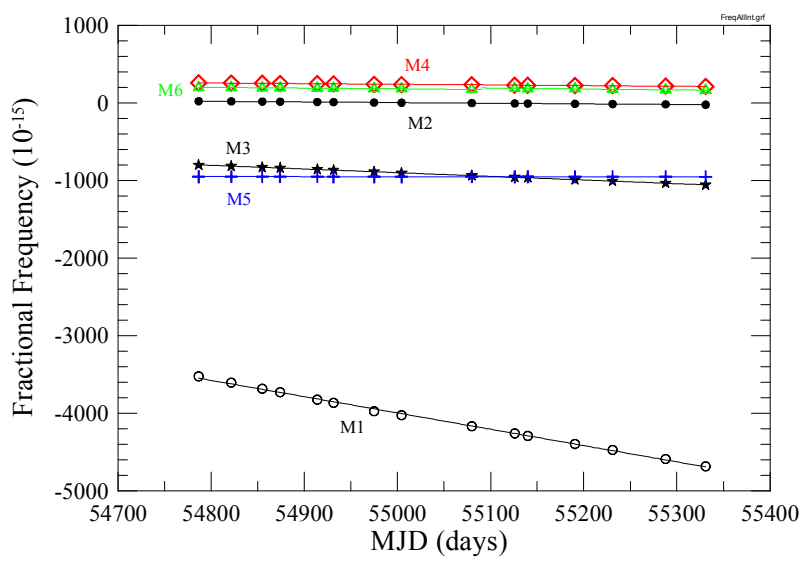

Figure 3. Internal maser frequencies relative to NIST-F1. An arbitrary and identical frequency offset has been applied to all masers. The solid lines are linear least mean square fits. 


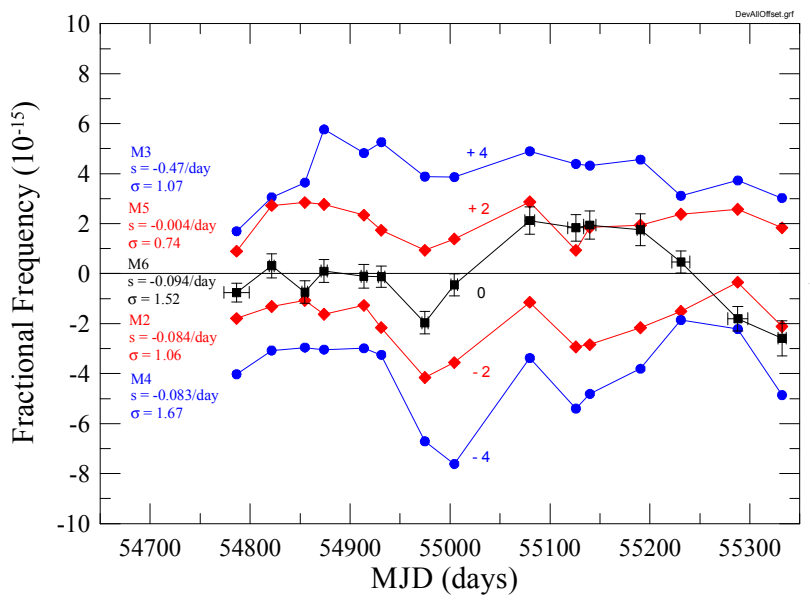

Figure 4. Deviations of maser frequencies about the linear fits as measured by NIST-F1. Average slopes, s, (in units of $10^{-15} /$ day) and standard deviations, $\sigma$, (in units of $10^{-15}$ ) are also shown.

These are shown in Fig. 4. Data from masers M2 through M6 are shown (M1 will be discussed later) and the curves have been offset along the vertical axis by amounts ranging from -4 $\mathrm{x} 10^{-15}$ to $+4 \times 10^{-15}$ for visual clarity. In addition to the deviation curves, the slope, $\mathrm{s}$, in units of $10^{-15}$ per day, and standard deviations, $\sigma$, in units of $10^{-15}$, for the fits are also shown in the figure. The vertical error bars shown on the M6 data represent the uncertainty of NIST-F1 for each measurement, and the horizontal error bars represent the duration of the measurement. The same error bars apply to all the masers, but are shown only for M6.

The feature in Fig. 4 that stands out the most is that there is a common slow frequency deviation characteristic for all of the masers during the first two thirds of the measurement interval. The magnitude of the dip in frequency that occurs around MJD 55000 varies in amplitude among the masers, but is present to some degree in all of the maser frequencies. The correlation among the masers is not as strong in the latter third of the measurement interval.

One possible cause for the common shape to the deviations would be frequency fluctuations in the reference standard. However, the deviations present in Fig. 4 for some of the masers are far too large to be caused by NIST-F1 [3]. M4 shows a peak deviation on the order of $4 \times 10^{-15}$, which is about 8 times larger than the total uncertainty of NIST-F1. NIST-F1 has been compared extensively with other cesium fountain primary frequency standards, and there is no evidence that the stated uncertainties are incorrect [5]. Furthermore, if the common deviations were caused by NIST-F1, they would be approximately the same for all masers, which is not the case. A comparison of M4 with M3 using the time-scale data clearly shows the frequency dip near MJD 55000, and these data do not involve NIST-F1 at all. Finally, the dip on M4 can also be seen relative to UTC(USNO) (a completely independent time scale) via GPS common view and carrier phase. It is clear that NIST-F1 is not the source of the common structure seen Fig. 4.

Another possibility for the common signature is sensitivity to a common environmental disturbance. Although it is still too early to tell, it is possible that the dip near MJD 55000 is part of an annual event. Temperature and relative humidity are individually controlled and monitored for all the masers. Barometric pressure, magnetic field and power line voltage are all monitored, but not controlled. A careful examination of all the environmental data has shown no significant correlation between the frequency deviations and variations in the environmental parameters. The temperatures in individual chambers tend to be dominated by independent instabilities and generally do not reflect the fluctuations in the room temperatures. The rooms are both below ground level and do not exhibit any evidence of an annual cycle. In either case the temperature fluctuations in the rooms and in the chambers do not correlate with the frequency deviations.

Relative humidity in the rooms does show an annual cycle, peaking around early August (MJD 55050), but generally the individual chambers do not exhibit this cycle. Based on measured humidity sensitivities [2] and the magnitude of the humidity fluctuations, the observed frequency variations are too large. Also, the phase of the frequency variations leads the peak of humidity cycle by about 70 days (or lags the minimum by about 110 days). Thus humidity does not appear to be responsible for the observed frequency fluctuations.

Barometric pressure variations also show an annual term, but are too small based on measured sensitivities and, like humidity, are also shifted in phase by about 70 days. Power line fluctuations are also too small. Magnetic field variations do not show the same characteristic as the frequency deviations. Thus none of the monitored environmental parameters appear to be responsible for the frequency variations.

If the dip near MJD 55000 appears again around 55365 this would be an indication of an annual event. In that case a more careful investigation of other environmental parameters will have to be made.

\section{B. Possible Frequency Coupling}

One observation that does show a strong correlation with

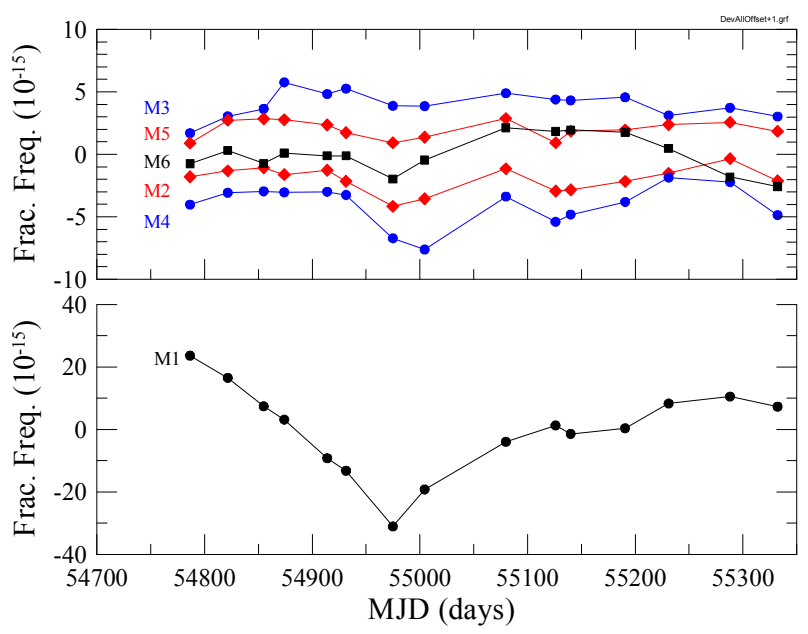

Figure 5. Comparison of the frequency deviations from a linear fit for maser M1 with the deviations of the other masers. 
the frequency deviations comes from maser M1. The top portion of Fig. 5 shows the same data as in Fig. 4, but Fig. 5 also includes in the lower portion the frequency deviations from the linear fit for maser M1. Note the difference in the vertical scales. The change in slope of the M1 frequency coincides with a sudden $3 \%$ drop in the discharge current of the $110 \mathrm{MHz}$ oscillator in M1 that dissociates the hydrogen molecules in the source bulb. It is very clear that the cause for the change in current originated in the maser since there were increasing temperature and voltage changes for about 3 weeks leading up to the sudden decrease in current. After the current change the temperatures went back to normal, but some voltages stayed low. The data suggest that some component was gradually overheating for several weeks and then finally failed. It obviously could not have been a critical component since the maser is still operating, but it did affect the discharge current. Past experience has shown that a change in discharge current can affect the frequency drift rate. A specific cause for the change in discharge current has not yet been identified.

It is certainly possible that the timing of the large change in drift rate in maser M1 and the changes in all the other masers is purely a coincidence. However, the strong correlation raises the possibility that there is some kind of frequency coupling between the masers. Note the converging of the frequencies of M2 and M4 in Fig. 1. The coupling may not involve the maser frequencies directly, but may be some mechanism by which power from the $110 \mathrm{MHz}$ source bulb oscillator (which dissipates about 2 watts) is leaking into the source bulb circuitry of the other masers and affecting the frequencies. However, these oscillators appear to be well shielded. It is also possible that what we are seeing is some common response in all the masers to an external environmental parameter that is not being monitored.

An additional piece of information that is relevant to the question of frequency coupling is shown in Fig. 6. During the course of this investigation maser M6 had to be physically moved a small amount for some routine maintenance. Because of this disturbance the frequency jumped upwards by about $1.5 \times 10^{-14}$. This step was removed from the data in Fig. 4, but is shown here in Fig. 6. There was no evidence from

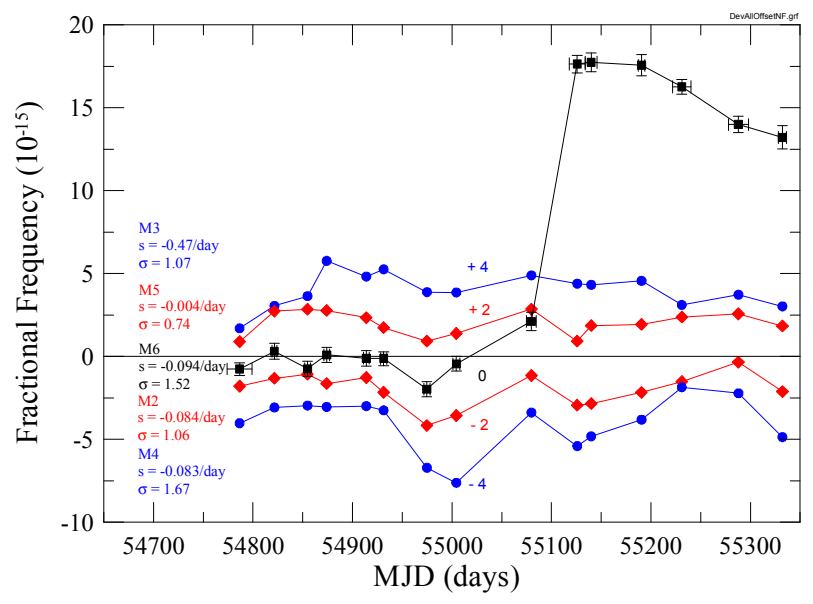

Figure 6. Frequency deviations with the step in frequency in M6 not removed. the internal data logged by the maser as to what had caused the frequency step. Coincident with the upward step in M6 there are also small downward frequency steps all the other masers. These are all small steps that are within the range of the random fluctuations, so it could be a coincidence, but it could also be another indication of frequency pulling. In any case, the frequency step in M6 did not cause any large frequency steps in the other masers.

The masers are all connected to the time scale measurement system via coaxial cables so there is also the possibility that this could be a source of frequency coupling. However, the $5 \mathrm{MHz}$ outputs of all the masers are connected to the measurement system through multiple high isolation distribution amplifiers. The $100 \mathrm{MHz}$ output of maser M2 is connected directly to NIST-F1 through a high isolation distribution amplifier and the same frequency deviations as shown in Fig. 4 are seen in the data measured directly by NIST-F1. The time scale measurement system is not involved at all in these direct measurements between NIST-F1 and M2.

At this time the data are ambiguous as to whether there is any frequency coupling between the masers. Future tests are planned in which the discharge current of M1 will be deliberately changed to see whether this affects the frequencies of the other masers.

\section{FREQUENCY DRIFT RATES}

The original goal of this investigation was to quantify the frequency drift rates of the masers and this has been accomplished. Figure 7 shows a typical plot of the frequency drift rate as a function of time for maser M2. Individual measurements are shown as black dots and a three point running average is shown as red diamonds. The error bars indicate the uncertainty of the measurements. For this maser the average drift rate is about $0.8 \times 10^{-16} / \mathrm{d}$, but peak to peak variations are almost $+/-50 \%$. The large excursions around MJD 55000 of course correspond to the dip in frequency seen in Fig. 4 around the same time.

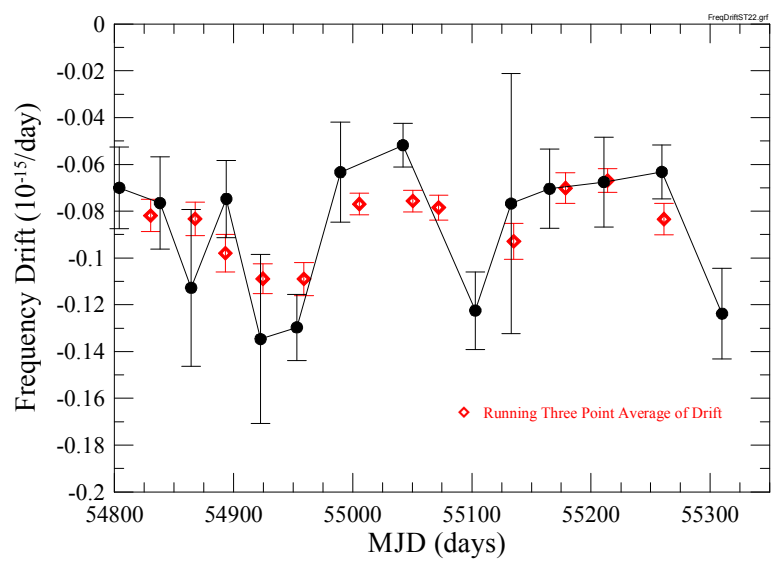

Figure 7. Frequency drift rate of M2. The red diamonds represent a three-point running average. 
Table 1 Maser Frequency Drift Rates

\begin{tabular}{|c|c|c|c|}
\hline Maser & $\begin{array}{c}\text { Avg. Freq. Drift } \\
10^{-16} / \mathrm{d}\end{array}$ & $\begin{array}{c}\text { Std. } \\
\text { Dev. } \\
10^{-16} / \mathrm{d}\end{array}$ & $\begin{array}{c}\text { RMS Time Prediction } \\
\text { Error at 30 days (ns) }\end{array}$ \\
\hline M1 & -21.07 & 2.45 & 9.5 \\
\hline M2 & -0.84 & 0.28 & 1.1 \\
\hline M3 & -4.72 & 0.38 & 1.5 \\
\hline M4 & -0.82 & 0.39 & 1.5 \\
\hline M5 & -0.04 & 0.30 & 1.2 \\
\hline M6 & -0.92 & 0.32 & 1.1 \\
\hline
\end{tabular}

Table 1 summarizes the maser drift rates. Columns 2 gives the average drift rate over the entire test interval and column 3 is the standard deviation about this mean. Maser 1 of course has the highest drift rate because of the repairs. All of the other masers have more normal drift rates. These linear rates range over more than two orders of magnitude because almost all masers go through a zero drift rate sometime in their operating life. However, the standard deviations are all roughly in the range of 3 to $4 \times 10^{-17} /$ day. The RMS time prediction errors at 30 days due to deviations in drift rates are shown in the fourth column. The time prediction error is calculated from Eq. 1, where E is the RMS time error, D is the standard deviation of the drift rate and $t=30$ days. Except for maser M1, the peak-to-peak time prediction errors are on the order of 3 to 4 nanoseconds. Of course other factors enter into the performance of a time scale, but errors just due to drift rates errors will be on the order of a few nanoseconds if the maser frequencies can be accurately measured about once a month.

$$
E=\frac{1}{2} D t^{2}
$$

\section{ALLAN DEVIATIONS}

The data obtained in this investigation also allow the Allan deviation (ADEV) of the masers to be estimated for $\tau$ values in the range of 40 to 200 days. The ADEV can only be estimated because the data are not evenly spaced and there is also significant dead time. For these calculations the data were treated as if they were evenly spaced. The TOTAL method was used to obtain the best estimate at large values of $\tau$. Figure 8 shows the highest and lowest estimated ADEV curves for the masers (M4 and M2 respectively), excluding M1. The curves with the black diamonds or dots are calculated from the data as they are, and the blue curves with hollow diamond or circles are corrected for dead time using the Barnes bias tables for flicker frequency noise [6]. The software package used to calculate ADEV only calculates confidence limits for octave intervals of $\tau$, so some $\tau$ values show no confidence limits.

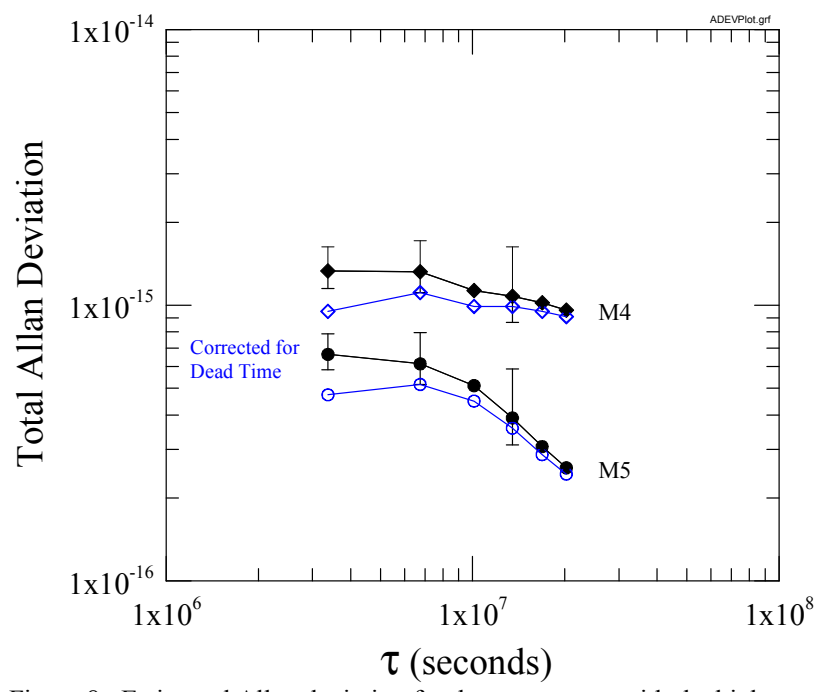

Figure 8. Estimated Allan deviation for the two masers with the highest and lowest ADEV values.

The frequency instabilities are roughly flicker in nature (as expected) and range from approximately $5 \times 10^{-16}$ to $1 \times 10^{-15}$. The roll-off at large $\tau$ occurs because the ADEV calculations were performed on data with linear frequency drift removed. The average type A uncertainty of NIST-F1 was about $3.7 \times 10^{-16}$, so it makes a small contribution to the ADEV values. The ADEV values in Fig. 8 are somewhat larger than those obtained from timescale data and this is to be expected because we have observed some degree of correlation in the maser frequency fluctuations.

\section{SUMMARY}

The operation of NIST-F1 on a nearly monthly basis (in combination with the NIST timescale measurement system) has allowed an unprecedented measurement of the frequency stability of six hydrogen masers at time intervals ranging from less than 40 days out to about 200 days. In these measurements we have observed a moderate level of correlation in the frequency fluctuations of the masers. Over a particular 100 day interval of time an average (averaged over five masers) peak common frequency deviation from linear drift of about $2 \times 10^{-15}$ occurred. A consequence of this correlation is that the assumption that the stability of the NIST time scale is improved by one over the square root of the number of masers is not justified. At this time the source of the correlation has not been identified. It does not come from NIST-F1, and none of the monitored environmental parameters has been identified as a source. There is some evidence that there may be some coupling between the masers, but this has yet to be confirmed. It is also possible that there is an as of yet unidentified common environmental sensitivity. More experiments are planned for the near future to identify the source.

\section{REFERENCES}

[1] T.E. Parker, "Hydrogen maser ensemble performance and characterization of frequency standards," in Proc. of the 1999 Joint Meeting of the IEEE International Frequency Control Symp. and the European Frequency and Time Forum, pp. 173-176, 1999. 
[2] T.E. Parker, "Environmental factors and hydrogen maser frequency stability," IEEE Trans. on Ultrasonics, Ferroelectrics, and Frequency Control, vol. 46, no. 3, pp.745-751, 1999.

[3] T.P. Heavner, S.R. Jefferts, E.A. Donley, J.H. Shirley, and T.E. Parker, "NIST-F1: Recent Improvements and Accuracy Evaluations," Metologia, 42, pp 411-422, 2005.

[4] N. Ashby, T.P. Heavner, S.R. Jefferts, T.E. Parker, A.G. Radnaev and Y.O Dudin, "Testing Local Position Invariance with Four Cesium-
Fountain Primary Frequency Standards and Four NIST Hydrogen Masers," Physical Review Letters, 98, 070802, 2007.

[5] T. E. Parker, "Long-term comparison of caesium fountain primary frequency standards," Metrologia, 47, pp1-10, 2010.

[6] J.A Barnes and D.W. Allan, "Variances Based on Data with Dead Time Between Measurements," NIST Technical Note 1318, pp. 1-40, 1990. 